\title{
Octet baryon magnetic moments at next-to-next-to-leading order in covariant chiral perturbation theory
}

\author{
Yang Xiao ${ }^{1}$, Xiu-Lei Ren ${ }^{2,3}$, Jun-Xu Lu ${ }^{1}$, Li-Sheng Geng ${ }^{4,5, a}$, Ulf-G. Meißner ${ }^{6,7}$ \\ ${ }^{1}$ School of Physics and Nuclear Energy Engineering, Beihang University, Beijing 100191, China \\ ${ }^{2}$ State Key Laboratory of Nuclear Physics and Technology, School of Physics, Peking University, Beijing 100871, China \\ ${ }^{3}$ Institut für Theoretische Physik II, Ruhr-Universität Bochum, 44780 Bochum, Germany \\ ${ }^{4}$ School of Physics and Nuclear Energy Engineering and Beijing Key Laboratory of Advanced Nuclear Materials and Physics, Beihang University, \\ Beijing 100191, China \\ ${ }^{5}$ China and Beijing Advanced Innovation Center for Big Date-based Precision Medicine, Beihang University, Beijing 100191, China \\ ${ }^{6}$ Helmholtz-Institut für Strahlen- und Kernphysik and Bethe Center for Theoretical Physics, Universität Bonn, 53115 Bonn, Germany \\ ${ }^{7}$ Institute for Advanced Simulation, Institut für Kernphysik and Jülich Center for Hadron Physics, Forschungszentrum Jülich, 52425 Jülich, \\ Germany
}

Received: 14 March 2018 / Accepted: 31 May 2018 / Published online: 12 June 2018

(C) The Author(s) 2018

\begin{abstract}
We calculate the octet baryon magnetic moments in covariant baryon chiral perturbation theory with the extended-on-mass-shell renormalization scheme up to nextto-next-to-leading order. At this order, there are nine lowenergy constants, which cannot be uniquely determined by the seven experimental data alone. We propose two strategies to circumvent this problem. First, we assume that chiral perturbation theory has a certain convergence rate and use this as one additional constraint to fix the low-energy constants by fitting to the experimental data. Second, we fit to lattice QCD simulations to determine the low-energy constants. We then compare the resulting predictions of the light and strange quark mass dependence of the octet baryon magnetic moments by the three mostly studied formulations of baryon chiral perturbation theory, namely, the extended-onmass-shell, the infrared, and the heavy baryon approaches. It is shown that once more precise lattice data become available, one will learn more about the convergence pattern of baryon chiral perturbation theory.
\end{abstract}

\section{Introduction}

SU(3) flavor symmetry and its breaking play an important role in our understanding of the strong interaction in the nonperturbative regime. In the limit of an exact SU(3) flavour symmetry, one can relate the magnetic moments of the octet baryons and the $\Lambda \Sigma^{0}$ transition to those of the proton and the neutron via the celebrated Coleman-Glashow formulae

\footnotetext{
a e-mail: lisheng.geng@buaa.edu.cn
}

[1]. Nonetheless, in nature $\mathrm{SU}(3)$ flavor symmetry is broken. This must be properly taken into account in order to improve the description of the baryon magnetic moments by inducing a realistic SU(3)-breaking mechanism. Chiral perturbation theory (ChPT), the low-energy effective field theory of QCD (see e.g. [2-6]), provides an appropriate framework to tackle this problem in a systematic fashion. However, it was noticed long ago that the leading order (LO) chiral corrections are large and tend to worsen the results, as exemplified, e.g., in [7-10]. This issue has often been used to question the validity of the SU(3) baryon ChPT altogether, see e.g. [11].

It was shown in Ref. [12] that one can achieve, however, an order by order improvement in the description of the octet baryon magnetic moments with the extended-on-mass-shell (EOMS) formulation of baryon ChPT $[13,14] .{ }^{1}$ Although it seems that the puzzle has been solved, a natural question is what happens at the next-to-next-to-leading order (NNLO). Because of the increased number of unknown low-energy constants (LECs), a clear answer to this question has not yet been provided.

In the last two decades several calculations of the octet baryon magnetic moments in heavy baryon (HB) ChPT up to NNLO have been performed both with $[9,18,19]$ and without [20] the inclusion of the baryon decuplet. It was shown in Ref. [20] that at NNLO the convergence of the HBChPT is quite good, contrary to the pattern exhibited at NLO. One

\footnotetext{
${ }^{1}$ Note that the contribution of the virtual decuplet baryons has a negligible effect on the overall description of the baryon magnetic moments [15], consistent with the heavy baryon (HB) findings of Ref. [16]. On the other hand, see Ref. [17] for discussions from a different perspective, focusing more on the contributions to individual baryons.
} 
should note that, however, in Ref. [20] the contributions of the two NNLO LECs $b_{6}^{D^{\prime}, F^{\prime}}$ are absorbed into the two LO ones $b_{6}^{D, F}$. This is legitimate as long as one works at the physical quark masses, as the primed LECs merely amount to a quark mass dependent shift of the unprimed ones that can not be disentangled.

Despite of all these studies, it remains unclear whether the convergence pattern of the HB [9], the infrared (IR) [10], and the EOMS formulation [12] observed in the description of the octet baryon magnetic moments, one of the cleanest observables, is particular to NLO and accidental, where no unknown LECs contribute, or it might be a more genuine feature of different formulations. Given that ChPT plays an indispensable role in our understanding of low energy strong interaction physics, it is of utmost importance to clarify this puzzling situation.

In this work we address this question by performing a study of the octet baryon magnetic moments at NNLO using the EOMS renormalization scheme. Since at this order the number of LECs is larger than that of the data, we first use convergence as a criterion to constrain the two LO LECs, $b_{6}^{D, F}$, which cannot be distinguished from the two NNLO LECs $b_{6}^{D^{\prime}, F^{\prime}}$ at the physical point. We then predict the light quark mass dependence of the octet baryon magnetic moments and contrast them with the results of the HB and IR formulations, and the state of the art lattice QCD simulations. Second, we fit the LECs to the lattice QCD data and then predict the strange quark mass dependence of the magnetic moments. It is shown that depending on how one determines the LECs, the predicted dependence is rather different, which could be investigated in more detail by future lattice QCD simulations.

This paper is organized as follows. In Sect. 2, we present the theoretical framework. Results and discussions are given in Sect. 3, followed by a short summary in Sect. 4.

\section{Theoretical framework}

The octet baryon magnetic moments are defined via baryon matrix elements of the electromagnetic current $J_{\mu}$ as follows

$$
\left\langle\bar{B}\left|J_{\mu}\right| B\right\rangle=\bar{u}_{B}\left(p_{f}\right)\left[\gamma_{\mu} F_{1}^{B}(t)+\frac{i \sigma_{\mu \nu} q^{\nu}}{2 m} F_{2}^{B}(t)\right] u_{B}\left(p_{i}\right),
$$

where $\bar{u}_{B}$ and $u_{B}$ are Dirac spinors, $m$ is the baryon mass, and $F_{1}^{B}$ and $F_{2}^{B}$ denote the Dirac and Pauli form factor, respectively. The four-momentum transfer is defined as $q=p_{f}-p_{i}$ and $t=q^{2}$. At $t=0, F_{2}^{B}(0)$ is the so-called anomalous magnetic moment, $\kappa_{B}$, and the magnetic moment is $\mu_{B}=\kappa_{B}+q_{B}$, with $q_{B}$ the charge of the baryon.

In ChPT, one can calculate the baryon magnetic moments order by order, i.e.,
$\mu_{B}=\mu_{B}^{(2)}+\mu_{B}^{(3)}+\mu_{B}^{(4)}+\cdots$,

where the numbers in the superscripts are the chiral order, defined as $n_{\mathrm{ChPT}}=4 L-2 N_{M}-N_{B}+\sum_{k} k V_{k}$ for a properly renormalized diagram with $L$ loops, $N_{M}\left(N_{B}\right)$ meson (baryon) propagators, and $V_{k}$ vertices from the $k^{\text {th }}$ order Lagrangian. Because of the large non-zero baryon mass in the chiral limit, this power counting is broken in a naive application of the $\overline{\mathrm{MS}}$ regularization scheme [3]. To recover the power counting, several approaches have been proposed, such as the HB method [21,22], the IR approach [23] and the EOMS scheme $[13,14]$. In recent years, it has been shown that the EOMS scheme has some advantages because it satisfies all symmetry and analyticity constrains and converges relatively faster in certain cases, see e.g. Ref. [24] for a short review.

The Feynman diagrams needed to calculate $\mu_{B}$ up to NNLO are shown in Fig. 1. The LO contributions are provided by the following Lagrangian [25],

$\mathcal{L}_{M B}^{(2)}=\frac{b_{6}^{D}}{8 m_{0}}\left\langle\bar{B} \sigma^{\mu \nu}\left\{F_{\mu \nu}^{+}, B\right\}\right\rangle+\frac{b_{6}^{F}}{8 m_{0}}\left\langle\bar{B} \sigma^{\mu \nu}\left[F_{\mu \nu}^{+}, B\right]\right\rangle$,

where $\sigma^{\mu \nu}=\frac{i}{2}\left[\gamma^{\mu}, \gamma^{\nu}\right], F_{\mu \nu}^{+}=\left(u^{\dagger} Q F_{\mu \nu} u+u Q F_{\mu \nu} u^{\dagger}\right)$, $Q=|e| \operatorname{diag}(2,-1,-1) / 3$ is the quark charge matrix with $e$ the electron charge, $u=\exp \left[i \Phi / 2 F_{\phi}\right]$, with $\Phi$ the unimodular matrix containing the pseudoscalar nonet, $F_{\phi}$ the pseudoscalar decay constant, and $F_{\mu \nu}=\partial_{\mu} A_{\nu}-\partial_{\nu} A_{\mu}$ is the conventional photon field strength tensor. Moreover, $\langle\ldots\rangle$ denotes the trace in flavor space. At LO, there are only two LECs, $b_{6}^{D}$ and $b_{6}^{F}$.

The leading SU(3) breaking corrections come from loop diagrams. They arise at order $\mathcal{O}\left(p^{3}\right)$ in the chiral counting and are determined completely in terms of the lowest order LECs from $\mathcal{L}_{B}^{(1)}+\mathcal{L}_{M}^{(2)}+\mathcal{L}_{M B}^{(1)}$, namely,

$$
\begin{aligned}
\mathcal{L}_{B}^{(1)} & =\left\langle\bar{B} i \gamma^{\mu} D_{\mu} B-m_{0} \bar{B} B\right\rangle \\
\mathcal{L}_{M}^{(2)} & =\frac{F_{\phi}^{2}}{4}\left\langle u_{\mu} u^{\mu}+\chi^{+}\right\rangle, \\
\mathcal{L}_{M B}^{(1)} & =\frac{D}{2}\left\langle\bar{B} \gamma^{\mu} \gamma^{5}\left\{u_{\mu}, B\right\}\right\rangle+\frac{F}{2}\left\langle\bar{B} \gamma^{\mu} \gamma^{5}\left[u_{\mu}, B\right]\right\rangle,
\end{aligned}
$$

where $m_{0}$ denotes the baryon mass in the chiral limit, $D_{\mu} B=$ $\partial_{\mu} B+\left[\Gamma_{\mu}, B\right], \Gamma_{\mu}=\frac{1}{2}\left(u^{\dagger} \partial_{\mu} u+u \partial_{\mu} u^{\dagger}\right)-\frac{i}{2}\left(u^{\dagger} v_{\mu} u+\right.$ $\left.u v_{\mu} u^{\dagger}\right)$, with $v_{\mu}$ the vector source, $u_{\mu}=i\left(u^{\dagger} \partial_{\mu} u-\right.$ $\left.u \partial_{\mu} u^{\dagger}\right)+\left(u^{\dagger} v_{\mu} u-u v_{\mu} u^{\dagger}\right)$ and $\chi^{ \pm}=u^{\dagger} \chi u^{\dagger} \pm u \chi^{\dagger} u$. Here, $\chi=2 B_{0} \mathcal{M}$, with $\mathcal{M}$ the quark mass matrix $\mathcal{M}=$ $\operatorname{diag}\left(m_{l}, m_{l}, m_{s}\right)$. In what follows, we work in the isospin limit $m_{l}=\left(m_{u}+m_{d}\right) / 2$. Further, $B_{0}=|\langle 0|\bar{q} q| 0\rangle| / F_{\phi}^{2}$. The axial vector couplings $D$ and $F$ are determined from hyperon decays to be $D=0.8$ and $F=0.46$, and these values will be taken throughout. 
Fig. 1 Feynman diagrams contributing to the octet baryon magnetic moments up to NNLO. a Contributes to LO, while $\mathbf{b}$ and c depict the NLO corrections. All other diagrams represent the NNLO contribution. Solid, baryons, Goldstone bosons and photons, respectively. The small and medium solid dots refer to vertices obtained from $\mathcal{L}_{M B}^{(1)}$ and $\mathcal{L}_{B}^{(1)}$, in order. The heavy dots refer to vertices from $\mathcal{L}_{M B}^{(2)}$. The circles refer to vertices from $\mathcal{L}_{M}^{(2)}$. The crosses refer to mass insertions $\sim b_{D, F}$. The square refers to a vertex from $\mathcal{L}_{M B}^{(4)}$. The partners of the wave function renormalization $(\mathbf{g})$ and baryon mass insertion (i) are not shown explicitly dashed and wiggly lines denote

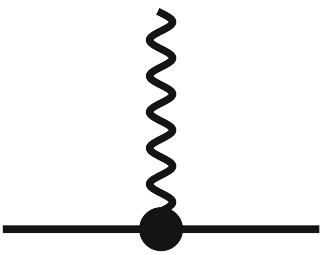

(a)

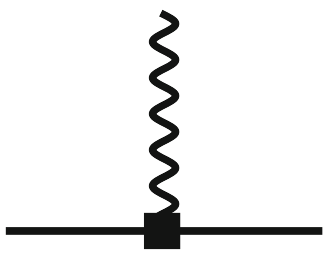

(d)

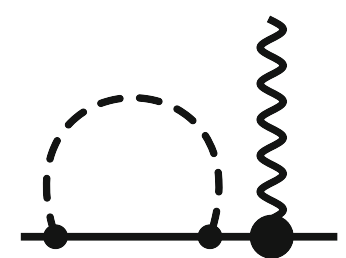

(g)

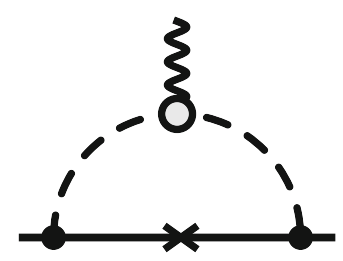

(j)

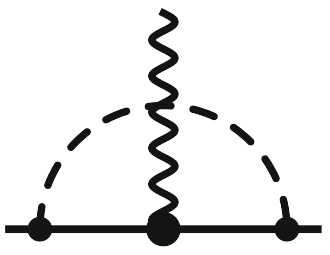

(b)

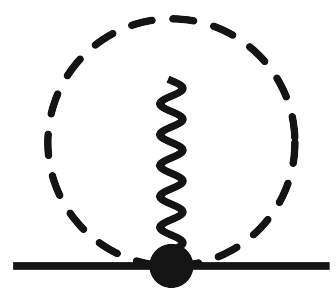

(e)

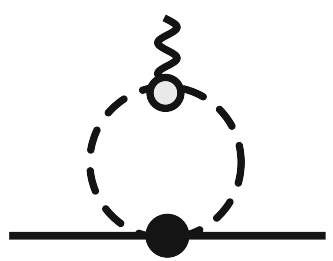

(h)

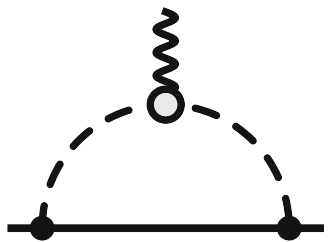

(c)

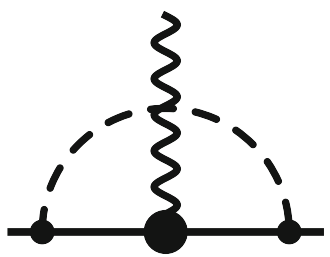

(f)

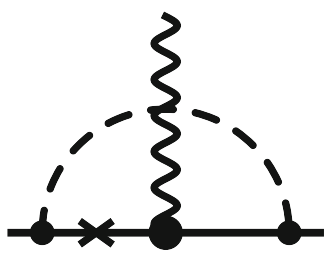

(i)
The explicit expressions of the LO and NLO results can be found in Ref. [12]. In the following, we focus on the NNLO contributions. At this order one has to include oneloop diagrams with exactly one vertex from $\mathcal{L}_{M B}^{(2)}$ as well as additional tree contributions from $\mathcal{L}_{M B}^{(4)}$. The fourth order contribution to $\mu_{B}$ is given as (see Fig. 1):

$$
\begin{gathered}
\mu^{(4)}=\mu^{(4, d)}+\mu^{(4, e)}+\mu^{(4, f)}+\mu^{(4, g)} \\
+\mu^{(4, h)}+\mu^{(4, i)}+\mu^{(4, j)} .
\end{gathered}
$$

The terms contributing to $\mu_{B}^{(4, d)}$ collect the tree contributions with exactly one insertion from the following chiral Lagrangian $[20,26]$,

$$
\begin{aligned}
\mathcal{L}_{M B}^{(4)}= & +\frac{b_{6}^{D^{\prime}}}{8 m_{0}}\left\langle\chi^{+}\right\rangle\left\langle\bar{B} \sigma^{\mu \nu}\left\{F_{\mu \nu}^{+}, B\right\}\right\rangle \\
& +\frac{b_{6}^{F^{\prime}}}{8 m_{0}}\left\langle\chi^{+}\right\rangle\left\langle\bar{B} \sigma^{\mu \nu}\left[F_{\mu \nu}^{+}, B\right]\right\rangle \\
& +\frac{\alpha_{1}}{8 m_{0}}\left\langle\bar{B} \sigma^{\mu \nu}\left[\left[F_{\mu \nu}^{+}, B\right], \chi^{+}\right]\right\rangle \\
& +\frac{\alpha_{2}}{8 m_{0}}\left\langle\bar{B} \sigma^{\mu \nu}\left\{\left[F_{\mu \nu}^{+}, B\right], \chi^{+}\right\}\right\rangle
\end{aligned}
$$

where $b_{6}^{D^{\prime}}, b_{6}^{F^{\prime}}, \alpha_{1,2,3,4}$ and $\beta_{1}$ are LECs.

At this order, we also have to consider double derivative operators at the meson-baryon vertex with the photon hooking on to the meson loop, see Fig. 1h. The corresponding terms of the dimension two Lagrangian read $[20,26]$

$\mathcal{L}_{M B}^{\left(2^{\prime}\right)}=\frac{i}{2}\left\{b_{9}\left\langle\bar{B} \sigma^{\mu \nu} u_{\mu}\right\rangle\left\langle u_{\nu} B\right\rangle+b_{10,11}\left\langle\bar{B} \sigma^{\mu v}\left(\left[u_{\mu}, u_{\nu}\right], B\right)_{ \pm}\right\rangle\right\}$,

where $b_{9,10,11}$ are LECs. They are estimated via resonance saturation in Ref. [20] and re-evaluated at $m_{0}=0.94 \mathrm{GeV}$, yielding $b_{9}=0.43 \mathrm{GeV}^{-1}, b_{10}=0.86 \mathrm{GeV}^{-1}$, and $b_{11}=$ $0.45 \mathrm{GeV}^{-1}$. We will call these values of the LECs set I. An improved determination has been given in Ref. [10], the corresponding values are $b_{9}=1.36 \mathrm{GeV}^{-1}, b_{10}=1.24$ 
$\mathrm{GeV}^{-1}$, and $b_{11}=0.46 \mathrm{GeV}^{-1}$. We will refer to these as set II.

At NNLO, one also needs the LO chiral corrections to the baryon masses, which are provided by the following Lagrangian [25]:

$\mathcal{L}_{M B}^{\left(2^{\prime \prime}\right)}=b_{D}\left\langle\bar{B}\left\{\chi_{+}, B\right\}\right\rangle+b_{F}\left\langle\bar{B}\left[\chi_{+}, B\right]\right\rangle$.

The two LECs $b_{D}$ and $b_{F}$ are fixed by fitting to the octet baryon mass splittings at $\mathcal{O}\left(p^{2}\right)$, yielding $b_{D}=$ $0.066 \mathrm{GeV}^{-1}$ and $b_{F}=-0.21 \mathrm{GeV}^{-1}$.

The NNLO tree-level contributions can be obtained rather straightforwardly. The results are shown in the Appendix. Following the EOMS prescription to restore the power counting, we obtain the following NNLO loop results,

$$
\begin{aligned}
\mu_{B}^{(4, e)}\left(M_{\phi}\right)= & C_{B}^{(4, e)}(\phi) \frac{M_{\phi}^{2}}{16 \pi^{2} F_{\phi}^{2}} \log \left(\frac{M_{\phi}^{2}}{\lambda^{2}}\right) \\
\mu_{B}^{(4, f)}\left(M_{\phi}\right)= & \frac{-C_{B}^{(4, f)}(\phi)}{16 \pi^{2} F_{\phi}^{2} m_{0}^{2} \sqrt{4 m_{0}^{2}-M_{\phi}^{2}}} \\
& \times\left\{\left(2 M_{\phi}^{5}-4 m_{0}^{2} M_{\phi}^{3}\right) \cos ^{-1}\left(\frac{M_{\phi}}{2 m_{0}}\right)\right. \\
& +\sqrt{4 m_{0}^{2}-M_{\phi}^{2}}\left\{m_{0}^{2} M_{\phi}^{2}\left[\log \left(\frac{M_{\phi}^{2}}{\lambda^{2}}\right)+2\right]\right. \\
& \left.\left.-M_{\phi}^{4} \log \left(\frac{M_{\phi}^{2}}{m_{0}^{2}}\right)\right\}\right\}, \\
\mu_{B}^{(4, g)}\left(M_{\phi}\right)= & \frac{C_{B}^{(4, g)}(\phi) M_{\phi}^{2}}{16 \pi^{2} F_{\phi}^{2} m_{0}^{2}\left(4 m_{0}^{2}-M_{\phi}^{2}\right)} \\
& \times\left\{( 4 m _ { 0 } ^ { 2 } - M _ { \phi } ^ { 2 } ) \left\{m_{0}^{2}\left[\log \left(\frac{\lambda^{2}}{m_{0}^{2}}\right)-4\right]\right.\right. \\
& \left.+\left(2 M_{\phi}^{2}-3 m_{0}^{2}\right) \log \left(\frac{M_{\phi}^{2}}{m_{0}^{2}}\right)\right\} \\
& \left.+4 M_{\phi}\left(3 m_{0}^{2}-M_{\phi}^{2}\right) \sqrt{4 m_{0}^{2}-M_{\phi}^{2}} \cos ^{-1}\left(\frac{M_{\phi}}{2 m_{0}}\right)\right\},
\end{aligned}
$$

$$
\begin{aligned}
\mu_{B}^{(4, h)}\left(M_{\phi}\right)= & C_{B}^{(4, h)}(\phi) \frac{m_{0} M_{\phi}^{2} \log \left(\frac{M_{\phi}^{2}}{\lambda^{2}}\right)}{8 \pi^{2} F_{\phi}^{2}}, \\
\mu_{B}^{(4, i)}\left(M_{\phi}\right)= & \frac{C_{B}^{(4, i)}(\phi)}{4 \pi^{2} m_{0}^{3} F_{\phi}^{2}\left(4 m_{0}^{2}-M_{\phi}^{2}\right)^{3 / 2}} \\
& \times\left\{2 M_{\phi}\left(-16 m_{0}^{6}+30 m_{0}^{4} M_{\phi}^{2}-10 m_{0}^{2} M_{\phi}^{4}+M_{\phi}^{6}\right)\right. \\
& \times \cos ^{-1}\left(\frac{M_{\phi}}{2 m_{0}}\right) \\
& +\sqrt{4 m_{0}^{2}-M_{\phi}^{2}}\left[4 m_{0}^{6}-13 m_{0}^{4} M_{\phi}^{2}+2 m_{0}^{2} M_{\phi}^{4}\right.
\end{aligned}
$$

$$
\begin{aligned}
\mu_{B}^{(4, j)}\left(M_{\phi}\right)= & \frac{\left.\left.-\left(M_{\phi}^{3}-4 m_{0}^{2} M_{\phi}\right)^{2} \log \left(\frac{M_{\phi}^{2}}{m_{0}^{2}}\right)\right]\right\}}{4 \pi^{2} F_{\phi}^{2} m_{0}^{3}\left(4 m_{0}^{2}-M_{\phi}^{2}\right)^{3 / 2}} \\
& \times\left\{\sqrt { 4 m _ { 0 } ^ { 2 } - M _ { \phi } ^ { 2 } } \left[-20 m_{0}^{6}+15 m_{0}^{4} M_{\phi}^{2}-2 m_{0}^{2} M_{\phi}^{4}\right.\right. \\
& -\left(4 m_{0}^{6}-21 m_{0}^{4} M_{\phi}^{2}+9 m_{0}^{2} M_{\phi}^{4}-M_{\phi}^{6}\right) \\
& \left.\times \log \left(\frac{M_{\phi}^{2}}{m_{0}^{2}}\right)\right] \\
& -2 M_{\phi}\left(-32 m_{0}^{6}+37 m_{0}^{4} M_{\phi}^{2}-11 m_{0}^{2} M_{\phi}^{4}+M_{\phi}^{6}\right) \\
& \left.\times \cos ^{-1}\left(\frac{M_{\phi}}{2 m_{0}}\right)\right\}
\end{aligned}
$$

where the coefficients $C_{B}^{(4, e, f, h, i, j)}(\phi=\pi, K, \eta)$ are tabulated in the Appendix and $\lambda$ is the renormalization scale. We have checked that our results agree with those of Ref. [20] in the heavy mass limit up to analytical terms.

\section{Results and discussions}

At LO and NLO, the two LECs $b_{6}^{D, F}$ can be determined by fitting to the seven experimental data $\mu_{p, n, \Lambda, \Sigma^{+}, \Sigma^{-}, \Xi^{-}, \Xi^{0}}$. An extensive discussion of the EOMS results in comparison with the HB and IR results is given in Ref. [12]. At NNLO, however, there are nine LECs, two from the LO contribution and seven from the NNLO contribution. As a result, the experimental data alone can not uniquely determine all the nine LECs. In Ref. [20], the two NNLO LECs $b_{6}^{D^{\prime}, F^{\prime}}$ are absorbed by the two LO LECs, $b_{6}^{D, F}$, while in the present work we keep explicitly $b_{6}^{D^{\prime}, F^{\prime}}$. Note that at the physical point, only the combinations $\bar{b}_{6}^{D, F}=b_{6}^{D, F}+\left\langle\chi_{+}\right\rangle b_{6}^{D^{\prime}, F^{\prime}}$ are relevant. In our numerical analysis, the decay constant and the chiral limit value of the baryon masses are chosen to be $F_{\phi}=0.108 \mathrm{GeV}$, an average of the pion, kaon, and eta decay constants, and $m_{0}=0.94 \mathrm{GeV}$, following the argument in Ref. [20]. The dimensional regularization scale is set at $\lambda=1.0 \mathrm{GeV}$. We also have performed calculations allowing for slight variation of $m_{0}$ or $\lambda$ about these values, e.g., $\lambda=0.9 \sim 1.1 \mathrm{GeV}$ and $m_{0}=0.8 \sim 1.1 \mathrm{GeV}$, and found that such changes have negligible effects on our results. It should be noted that in principle at the order we are working, one should use the chiral limit values for $D, F$, and $F_{\phi}$. Using the physical/average values for them will lead to differences of higher chiral order. We checked that using the chiral limit values for $D, F$, and $F_{\phi}$, such as those determined in Ref. $[27,28]$, affect little our results and conclusions. 
Fig. 2 Left panel: dependence of the convergence rate $C_{R}=0.6$ on $b_{6}^{D, F}$ in the EOMS scheme. Right panel: convergence rates, $C_{R}$, of the $\mathrm{HB}$, IR, and EOMS scheme as a function of $b_{6}^{D, F}$. The green shaded area in both plots are the same. The blue stars denote the optimal $b_{6}^{D, F}$, located in the center of the shaded area and later used to study convergence, i.e.,

$\left(b_{6}^{D}, b_{6}^{F}\right)=(3.54,1.17)_{\mathrm{EOMS}}$, $(4.22,2.30)_{\mathrm{HB}},(3.96,1.00)_{\mathrm{IR}}$

Table 1 Contributions of different chiral orders of the HB, IR, and EOMS schemes up to NNLO with the LECs of Table 2
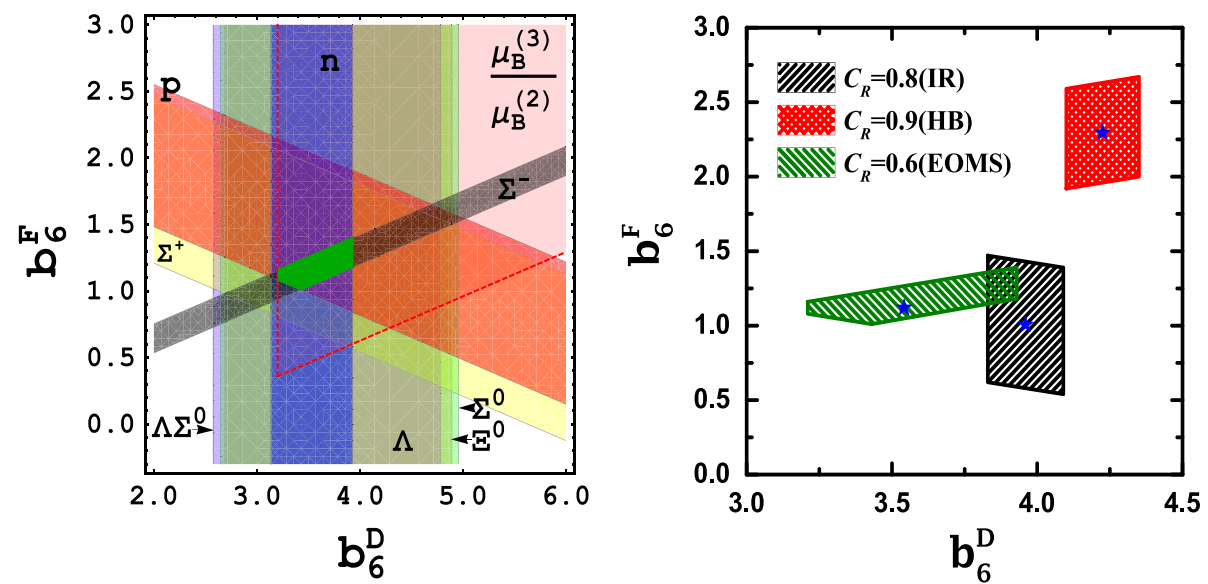

\begin{tabular}{|c|c|c|c|c|c|c|}
\hline \multirow[t]{2}{*}{ Baryons } & \multicolumn{2}{|l|}{ EOMS } & \multicolumn{2}{|l|}{ IR } & \multicolumn{2}{|l|}{ HB } \\
\hline & $\mu_{B}^{(3)} / \mu_{B}^{(2)}$ & $\mu_{B}^{(4)} / \mu_{B}^{(3)}$ & $\mu_{B}^{(3)} / \mu_{B}^{(2)}$ & $\mu_{B}^{(4)} / \mu_{B}^{(3)}$ & $\mu_{B}^{(3)} / \mu_{B}^{(2)}$ & $\mu_{B}^{(4)} / \mu_{B}^{(3)}$ \\
\hline$p$ & -0.27 & -0.38 & -0.16 & 0.01 & -0.44 & -0.07 \\
\hline$n$ & -0.19 & 0.02 & -0.17 & 0.61 & -0.18 & 0.74 \\
\hline$\Lambda$ & -0.52 & -0.08 & -0.73 & -0.27 & -0.83 & 0.32 \\
\hline$\Sigma^{-}$ & 0.18 & -0.04 & 2.58 & -0.73 & -0.30 & 0.30 \\
\hline$\Sigma^{+}$ & -0.31 & -0.15 & -0.05 & 4.20 & -0.61 & -0.22 \\
\hline$\Sigma^{0}$ & -0.52 & -0.13 & -0.73 & -0.31 & -0.83 & -0.35 \\
\hline$\Xi^{-}$ & 0.03 & -12.88 & 3.10 & -1.02 & -0.74 & -0.12 \\
\hline$\Xi^{0}$ & -0.54 & -0.13 & -0.77 & -0.32 & -0.87 & -0.36 \\
\hline$\Lambda \Sigma^{0}$ & -0.31 & 0.27 & -0.38 & -0.11 & -0.43 & 0.46 \\
\hline
\end{tabular}

Table 2 LO and NNLO low-energy constants denoted by the blue stars in Fig. 2, where $\bar{b}_{6}^{D}$ and $\bar{b}_{6}^{F}$ are linear combinations of the two LO and NNLO LECs, $\bar{b}_{6}^{D}=b_{6}^{D}+\left\langle\chi_{+}\right\rangle b_{6}^{D^{\prime}}, \bar{b}_{6}^{F}=b_{6}^{F}+\left\langle\chi_{+}\right\rangle b_{6}^{F^{\prime}}$

\begin{tabular}{lccccccccccc}
\hline Chiral Schemes & $b_{6}^{D}$ & $b_{6}^{F}$ & \multicolumn{1}{c}{$\alpha_{1}$} & \multicolumn{1}{c}{$\alpha_{2}$} & $\alpha_{3}$ & $\alpha_{4}$ & $\beta_{1}$ & $b_{6}^{D^{\prime}}$ & $b_{6}^{F^{\prime}}$ & $\bar{b}_{6}^{D}$ & $\bar{b}_{6}^{F}$ \\
\hline IR & 3.96 & 1.00 & -0.89 & -0.57 & -0.19 & 0.41 & -2.98 & -3.85 & -1.21 & 0.04 & -0.23 \\
HB & 4.22 & 2.30 & -1.49 & 0.07 & -1.27 & 1.74 & -2.41 & -2.79 & -0.82 & 1.38 & 1.46 \\
EOMS & 3.54 & 1.17 & 0.08 & 0.48 & 0.08 & 0.83 & -0.90 & -1.69 & -0.21 & 1.82 & 0.95 \\
\hline
\end{tabular}

\subsection{Using convergence to constrain the low-energy} constants

We assume that $\mathrm{BChPT}$ has a reasonable convergence rate in the $u, d, s$ three flavor sector, namely, higher order contributions are suppressed compared to lower order ones, in terms of $M_{K} / \Lambda_{\mathrm{ChPT}} \approx 0.5$. Under this assumption, we can use the convergence criterion and the experimental data to constrain the nine LECs. More specifically, we define the convergence rate $\left(C_{R}\right)$ as

$$
\begin{aligned}
C_{R} & =\max \left(\mu_{B}^{(3)} / \mu_{B}^{(2)}, \mu_{B}^{(4)} / \mu_{B}^{(3)}\right) \quad \text { with } \\
B & =p, n, \Lambda, \Sigma^{+}, \Sigma^{0}, \Sigma^{-}, \Lambda \Sigma^{0}, \Xi^{-}, \Xi^{0} .
\end{aligned}
$$

When fitting, we can set the convergence rate to a particular value, e.g., 0.5, and search for a combination of LECs that can satisfy such a requirement. In the left panel of Fig. 2, we show how one can fix the range of $b_{6}^{D, F}$ by requiring $C_{R} \leq 0.6$ in the EOMS scheme. It can be seen that indeed there exist some combinations of $b_{6}^{D, F}$ which can satisfy the requirement, namely the green shaded area. In the right panel of Fig. 2, we show the best convergence rate achievable in the EOMS scheme, in comparison with those in the HB and IR schemes. One can see that only in the EOMS scheme a convergence rate about 0.6 can be achieved, ${ }^{2}$ while the convergence rates in the $\mathrm{HB}$ and IR schemes are relatively larger. This is consistent with the findings at NLO [12]. On the other

\footnotetext{
${ }^{2}$ Note that in the searches for the best convergence rate, in the EOMS scheme the $\Xi^{-}$channel is excluded because of its accidentally tiny contribution at NLO. In the same manner, in the IR scheme the $\Sigma^{ \pm}$and $\Xi^{-}$channels are also excluded .
} 

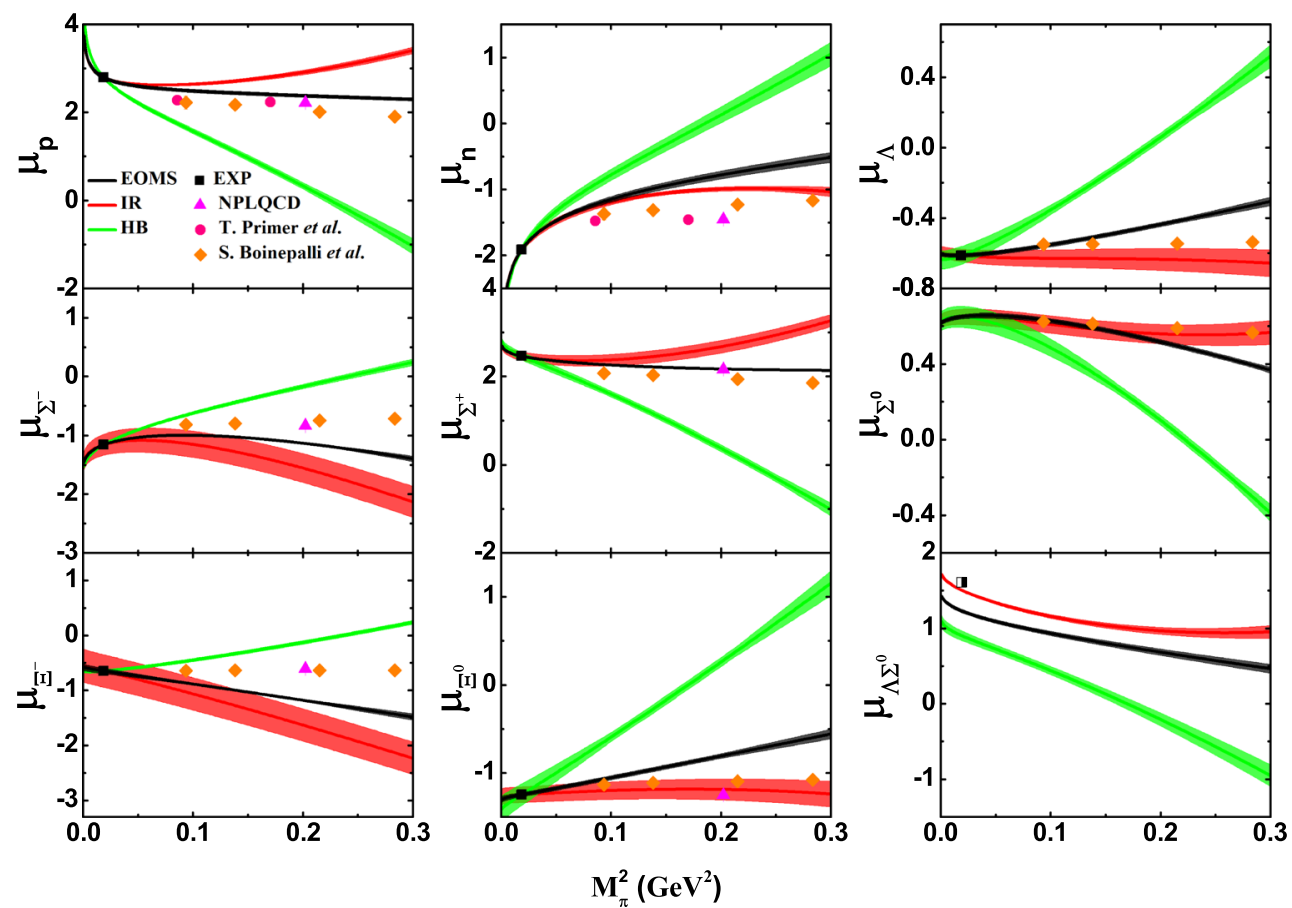

Fig. 3 Octet baryon magnetic moments as functions of the pion mass squared. The central values of the LECs are given in Table 2, while the shaded bands represent higher order

contributions not considered (see text for details). The half filled square indicates that we have chosen a positive sign for $\mu_{\Lambda \Sigma^{0}}$

Table $3 \tilde{\chi}^{2}$ between the ChPT results obtained in the HB, IR, and EOMS schemes and the LQCD data of Refs. [30-32] with the optimal $b_{6}^{(D, F)}$ denoted by the blue stars in Fig. 2

\begin{tabular}{llcl}
\hline Chiral schemes & $\tilde{\chi}^{2}$ & \\
\cline { 2 - 4 } & S. Boinepalli et al. [30] & NPLQCD [32] & T. Primer et al. [31] \\
\hline IR & 12.98 & 2.58 & 0.69 \\
HB & 43.45 & 12.5 & 4.64 \\
EOMS & 3.66 & 1.11 & 0.50 \\
\hline
\end{tabular}

hand, from a typical convergence pattern of the three renormalization schemes shown in Table 1 with the corresponding LECs given in Table 2, one notices that the contributions of different chiral orders in the HB ChPT are more moderate, though not as small as one naively expects.

It should be noted that in the numerical study we have taken the values given above for the LECs $D, F, b_{D}, b_{F}, b_{9}$, $b_{10}$, and $b_{11}$. In principle, there are some uncertainties. These can originate either from the data used to fix them, or from the chiral orders at which they are determined, or from the validity of the assumption adopted, such as resonance saturation for the case of $b_{9}, b_{10}$, and $b_{11}$. Unfortunately, there is no easy way to quantify these uncertainties. We have checked that using set II instead of set I, we find some quantitative differences but the overall trends are not affected.

One way to test the LECs determined above and also to distinguish different formulations of BChPT is to study the light quark mass dependence of the magnetic moments. Fixing the strange quark mass to its physical value with the LO ChPT relation $2 B_{0} m_{s}=\left.\left(2 M_{K}^{2}-M_{\pi}^{2}\right)\right|_{\text {phys. }}$, we show the
Table 4 Low-energy constants determined by fitting to the lattice QCD data of Ref. [30] and the corresponding $\tilde{\chi}^{2}$ in the IR, HB, and EOMS BChPT up to NLO

\begin{tabular}{lrrr}
\hline & \multicolumn{1}{c}{$b_{6}^{D}$} & \multicolumn{1}{c}{$b_{6}^{F}$} & \multicolumn{1}{c}{$\tilde{\chi}^{2}$} \\
\hline IR & 5.07 & -0.92 & 13.18 \\
HB & 5.29 & 2.95 & 5.75 \\
EOMS & 3.73 & 1.00 & 0.61 \\
\hline
\end{tabular}

pion mass dependence of the magnetic moments in Fig. 3. The lines are obtained with the LECs tabulated in Table 2, while the bands denote higher order contributions not considered in the NNLO study. They are obtained according to Ref. [29]:

$\delta \mu_{B}^{(i)}=\max \left(Q^{i-1}\left|\mu_{B}^{(2)}\right|, Q^{i+1-j}\left|\mu_{B}^{(j)}\right|\right), \quad 2 \leq j \leq i$,

where $\delta \mu_{B}^{(i)}$ are the uncertainties of chiral order $i, Q=$ $M_{\pi} / \Lambda_{\mathrm{ChPT}}, M_{\pi}=0.138 \mathrm{GeV}$, and $\Lambda_{\mathrm{ChPT}} \sim 1 \mathrm{GeV}$ is the chiral symmetry breaking scale. In addition, the requirement [29] 

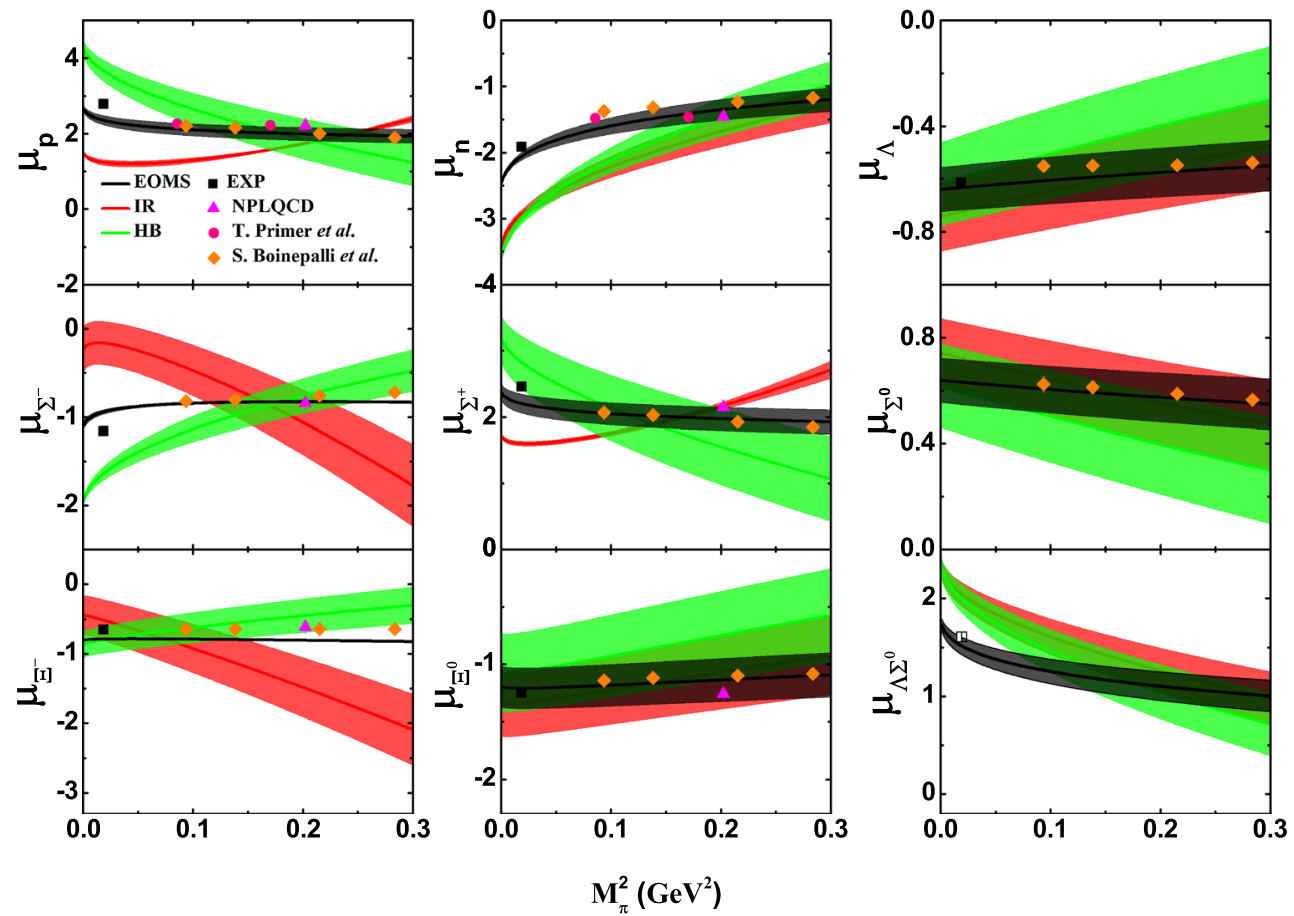

Fig. 4 Fits to the lattice QCD data of Ref. [30] at $\mathcal{O}\left(p^{3}\right)$ with the EOMS, HB, and IR BChPT. The bands represent estimated higher order corrections as explained in the text
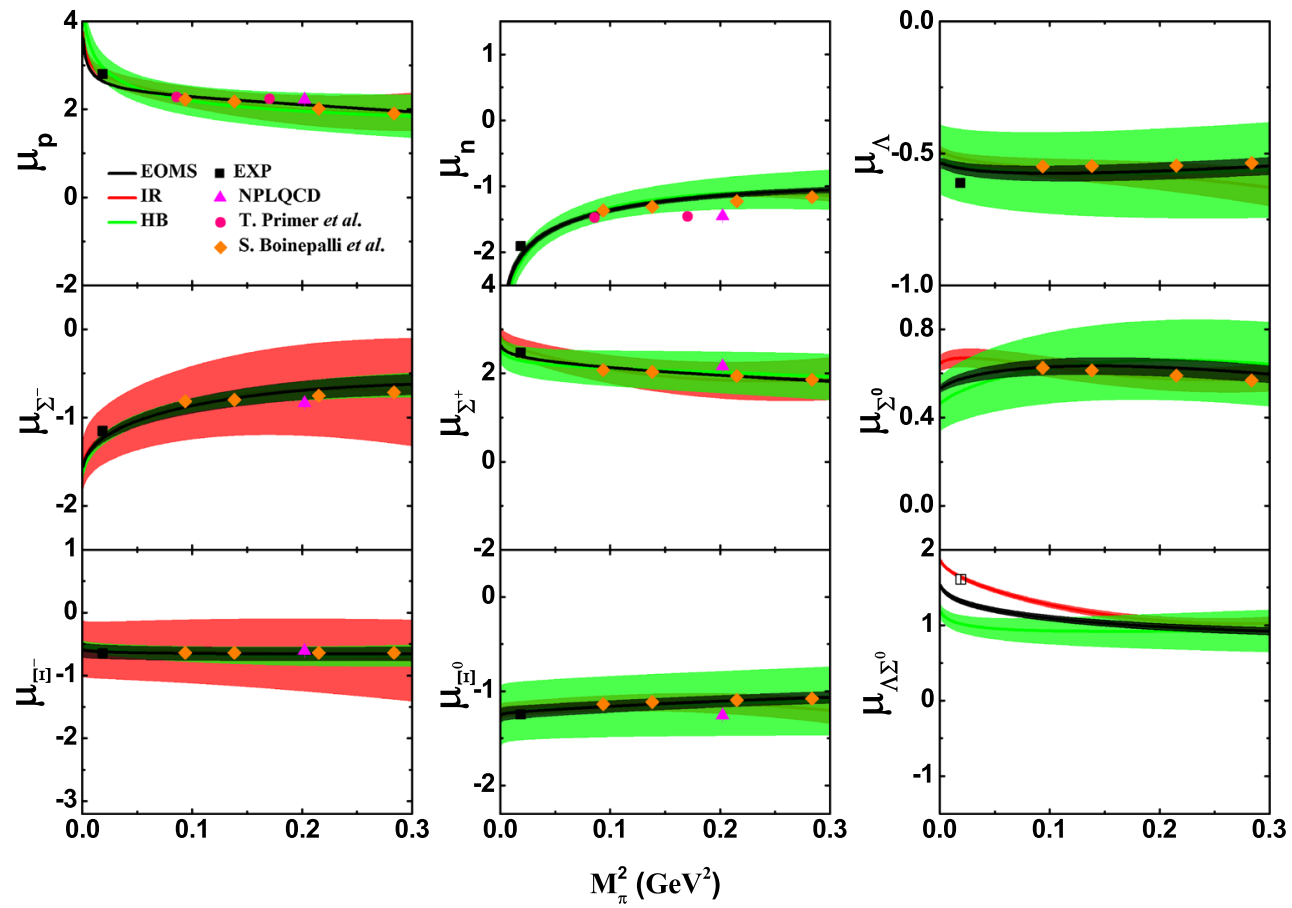

Fig. 5 Fits to the lattice QCD data of Ref. [30] at $\mathcal{O}\left(p^{4}\right)$ with the EOMS, HB, and IR BChPT. The bands represent higher order contributions as explained in the text

$\delta \mu_{B}^{(i+1)} \geq Q \delta \mu_{B}^{(i)}$

is also satisfied. It is clear that these contributions reflect partly the convergence pattern, i.e., the slower the convergence, the larger the higher order contributions.
One can see that the three schemes display rather different pion mass dependence. For comparison, the state of the art lattice QCD results [30-32] are shown as well. Note that such a comparison is only meant to be qualitative, since 
Table 5 Low-energy constants obtained by fitting to the lattice QCD data of Ref. [30] and the and EOMS BChPT up to NNLO

Table 6 Contributions of different chiral orders of the HB, IR, and EOMS schemes up to $\mathcal{O}\left(p^{4}\right)$ with the LECs obtained by fitting to the lattice QCD data corresponding $\tilde{\chi}^{2}$ in the IR, HB,

\begin{tabular}{lccccccccrc}
\hline & $b_{6}^{D}$ & $b_{6}^{F}$ & \multicolumn{1}{c}{$\alpha_{1}$} & \multicolumn{1}{c}{$\alpha_{2}$} & \multicolumn{1}{c}{$\alpha_{3}$} & $\alpha_{4}$ & $\beta_{1}$ & $b_{6}^{D^{\prime}}$ & \multicolumn{1}{c}{$b_{6}^{F^{\prime}}$} & $\tilde{\chi}^{2}$ \\
\hline IR & 4.02 & 2.08 & -0.20 & -0.83 & 0.06 & 0.20 & -2.88 & -3.66 & -3.59 & 0.14 \\
HB & 2.16 & 1.08 & -1.47 & 0.28 & -1.47 & 1.53 & -2.12 & 0.56 & 0.89 & 0.24 \\
EOMS & 3.03 & 1.40 & 0.17 & 0.30 & 0.15 & 0.60 & -0.56 & -0.69 & -0.59 & 0.13 \\
\hline
\end{tabular}

\begin{tabular}{|c|c|c|c|c|c|c|}
\hline \multirow[t]{2}{*}{ Baryons } & \multicolumn{2}{|l|}{ EOMS } & \multicolumn{2}{|l|}{ IR } & \multicolumn{2}{|l|}{ HB } \\
\hline & $\mu_{B}^{(3)} / \mu_{B}^{(2)}$ & $\mu_{B}^{(4)} / \mu_{B}^{(3)}$ & $\mu_{B}^{(3)} / \mu_{B}^{(2)}$ & $\mu_{B}^{(4)} / \mu_{B}^{(3)}$ & $\mu_{B}^{(3)} / \mu_{B}^{(2)}$ & $\mu_{B}^{(4)} / \mu_{B}^{(3)}$ \\
\hline$p$ & -0.26 & -0.12 & -0.12 & 2.09 & -0.73 & -1.08 \\
\hline$n$ & -0.22 & -1.09 & -0.17 & 0.38 & -0.35 & -2.36 \\
\hline$\Lambda$ & -0.61 & -0.26 & -0.72 & -0.15 & -1.62 & -0.85 \\
\hline$\Sigma^{-}$ & 0.13 & -1.85 & 1.02 & -1.30 & -0.41 & -0.81 \\
\hline$\Sigma^{+}$ & -0.31 & -0.01 & -0.04 & 10.21 & -1.03 & -0.82 \\
\hline$\Sigma^{0}$ & -0.61 & -0.29 & -0.72 & -0.31 & -1.62 & -0.82 \\
\hline$\Xi^{-}$ & 0.02 & -28.25 & 1.22 & -1.53 & -1.03 & -0.44 \\
\hline$\Xi^{0}$ & -0.64 & -0.38 & -0.76 & -0.27 & -1.71 & -0.91 \\
\hline$\Lambda \Sigma^{0}$ & -0.37 & -0.33 & -0.38 & -0.22 & -0.84 & -0.78 \\
\hline
\end{tabular}
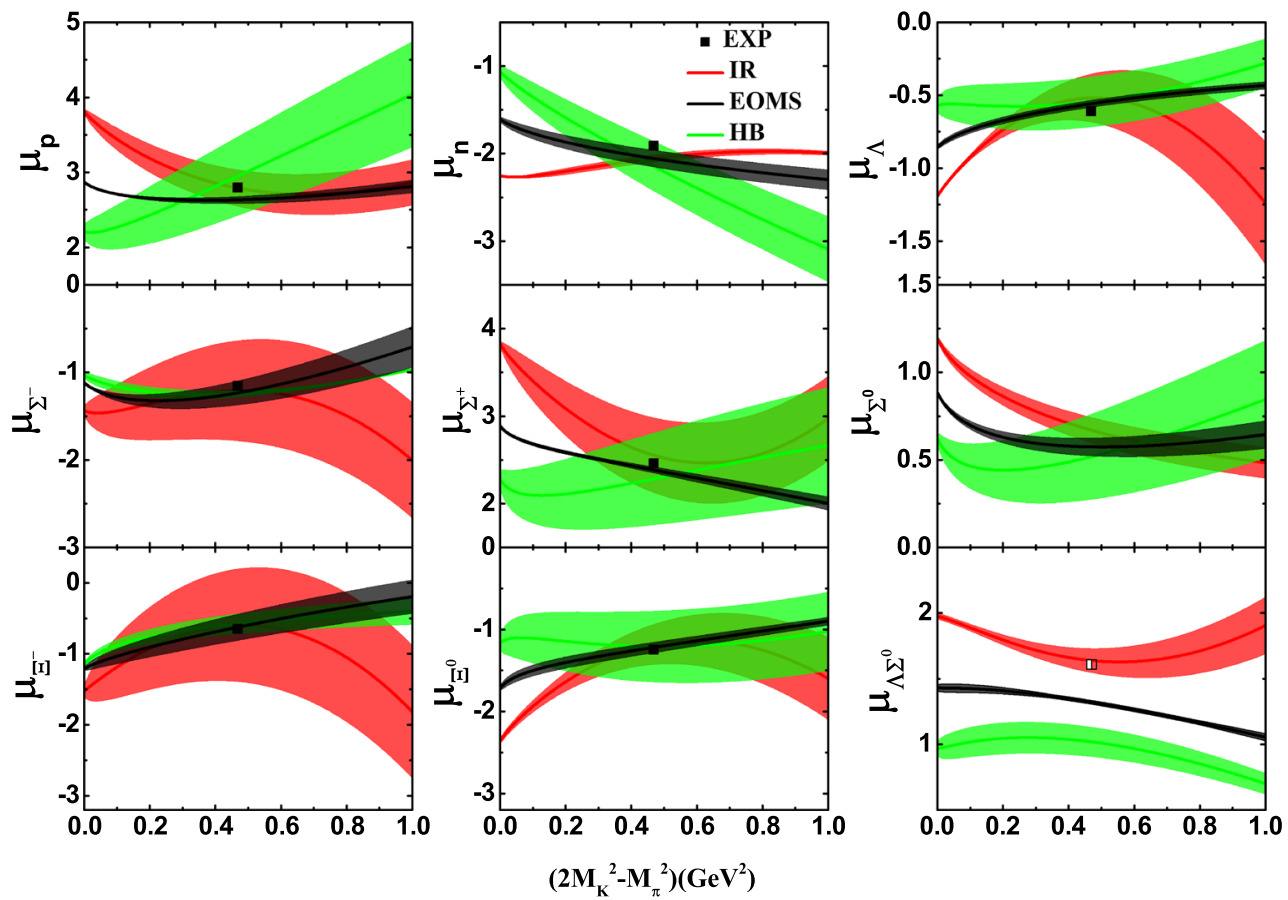

Fig. 6 Predicted dependence of the octet baryon magnetic moments on the strange quark mass with the LECs determined by fitting to the lattice QCD data of Ref. [30] at $\mathcal{O}\left(p^{4}\right)$ with the EOMS, HB, and IR

BChPT. The $u / d$ quark mass is fixed at their physical value while the strange quark mass is proportional to $2 M_{K}^{2}-M_{\pi}^{2}$ according to leading order ChPT. The bands represent higher order contributions 

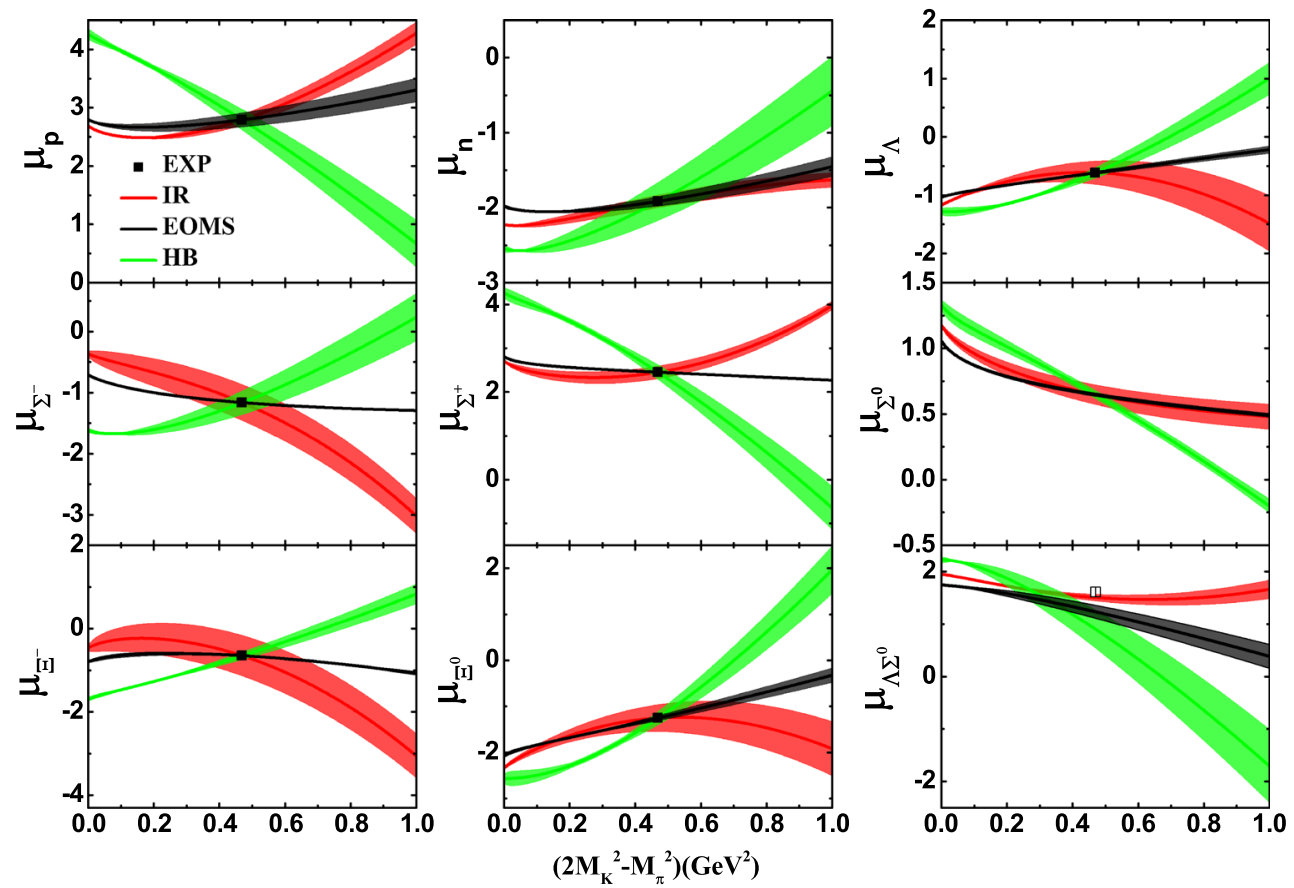

Fig. 7 The same as Fig. 6, but with the LECs given in Table 2

these simulations are performed with the strange quark mass close to its physical value but not exactly at the physical point and furthermore current lattice QCD simulations still contain systematic uncertainties not under complete control. It is clear that the EOMS results are in better agreement with the lattice data, as corroborated by the unweighted $\tilde{\chi}^{2}=\sum\left(\mu_{\text {th }}-\mu_{\text {lattice }}\right)^{2}$ between the results of each scheme and the lattice QCD data [30-32] shown in Table 3. One should note that only at relatively large pion masses, e.g., $M_{\pi}>200$ or $300 \mathrm{MeV}$, one can distinguish the results from different formulations of BChPT using lattice QCD simulations.

\subsection{Fitting to the lattice QCD data}

Now we take a more practical attitude, forgetting about the convergence constraint, and determine the relevant LECs by fitting to the lattice QCD data directly. Among the three lattice QCD studies we considered, the one of Ref. [30] features the largest number of simulation points. Therefore, we fit to these data to determine the LECs. At $\mathcal{O}\left(p^{3}\right)$, one has only two LECs. Their values from the best fit together with the corresponding $\tilde{\chi}^{2}$ are tabulated in Table 4 . The predicted pion mass dependence is shown in Fig. 4, where the lattice data from Refs. [31,32] are also shown. One can see that only the EOMS formulation can describe the lattice QCD data reasonably well, consistent with the finding in the SU(2) sector [33]. Furthermore, as shown in Table 4, the LECs of the EOMS formulation determined from the fit to the lattice QCD data are similar to those determined by the experimental data [12].

The situation becomes different, however, if we fit to the lattice data with the $\mathcal{O}\left(p^{4}\right) \mathrm{BChPT}$ results, all the three formulations can describe the lattice data with similar quality, as shown in Fig. 5 and Table 5. On the other hand, it seems that with the LECs determined from the best fit, the predicted $\mu_{\Lambda \Sigma_{0}}$ by the IR formulation is in better agreement with the experimental value (if a positive sign is taken). Nevertheless, one should note that the convergence pattern, particularly those of the IR and HB schemes, is destroyed (see Table 6).

One way to distinguish the different formulations in the present case is to study the strange quark mass dependence of the magnetic moments, shown in Figs. 6, and 7. One can see that depending on how one determines the LECs, either by fitting to the lattice QCD data or to the experimental data with the convergence constraint, the dependences on the strange quark mass are quite different. ${ }^{3}$ It is clear that one needs more lattice data with varying strange quark mass to check which scenario is more realistic.

\section{Summary}

We studied the octet baryon magnetic moments in baryon chiral perturbation theory with the extended-on-mass-shell

\footnotetext{
${ }^{3}$ We note that the EOMS predictions in the two cases are similar to each other.
} 
renormalization scheme up to next-to-next-to-leading order. We determined the low-energy constants following two different strategies, either by fitting to the experimental data with convergence as a further constraint or by fitting to lattice QCD data directly. It was shown that in the first case the extended-on-mass shell formulation seems to describe better the lattice QCD data, while in the second case, although all three formulations of baryon chiral perturbation theory can describe the lattice QCD data, they predict rather different strange quark mass dependence. Clearly more lattice QCD simulations are needed to better understand the situation and the convergence pattern of baryon chiral perturbation theory.

Acknowledgements This work is supported in part by the National Natural Science Foundation of China under Grants No. 11522539, No. 11735003, the China Postdoctoral Science Foundation (2016M600845, 2017T100008), and by DFG and NSFC through funds provided to the Sino-German CRC 110 "Symmetry and the Emergence of Structure in QCD” (NSFC Grant No. 11621131001, DFG Grant No. TRR110). The work of UGM was also supported by the CAS President's International Fellowship Initiative (PIFI) (Grant No. 2018DM0034).

Open Access This article is distributed under the terms of the Creative Commons Attribution 4.0 International License (http://creativecomm ons.org/licenses/by/4.0/), which permits unrestricted use, distribution, and reproduction in any medium, provided you give appropriate credit to the original author(s) and the source, provide a link to the Creative Commons license, and indicate if changes were made. Funded by $\mathrm{SCOAP}^{3}$.

\section{Appendix}

\section{A. NNLO tree level contributions}

Here, we list the tree level contributions at NNLO.

$$
\begin{aligned}
\mu_{p}^{(4, d)}= & \frac{2}{3}\left(3 b_{6}^{F^{\prime}}\left(2 M_{K}^{2}+M_{\pi}^{2}\right)+b_{6}^{D^{\prime}}\left(2 M_{K}^{2}+M_{\pi}^{2}\right)\right. \\
& +2\left(3 \alpha_{2} M_{K}^{2}+\alpha_{3} M_{K}^{2}+\alpha_{4} M_{K}^{2}-\beta_{1} M_{K}^{2}\right. \\
& \left.\left.+3 \alpha_{1}\left(M_{K}^{2}-M_{\pi}^{2}\right)-\alpha_{3} M_{\pi}^{2}+\beta_{1} M_{\pi}^{2}\right)\right), \\
\mu_{n}^{(4, d)}= & -\frac{4}{3}\left(b_{6}^{D^{\prime}}\left(2 M_{K}^{2}+M_{\pi}^{2}\right)+2 \alpha_{4} M_{K}^{2}\right. \\
& \left.+\beta_{1} M_{K}^{2}+2 \alpha_{3}\left(M_{K}^{2}-M_{\pi}^{2}\right)-\beta_{1} M_{\pi}^{2}\right), \\
\mu_{\Lambda}^{(4, d)}= & -\frac{2}{9}\left(3 b_{6}^{D^{\prime}}\left(2 M_{K}^{2}+M_{\pi}^{2}\right)\right. \\
& \left.+2\left(\alpha_{4}\left(8 M_{K}^{2}-5 M_{\pi}^{2}\right)+3 \beta_{1}\left(M_{K}^{2}-M_{\pi}^{2}\right)\right)\right), \\
\mu_{\Sigma^{+}}^{(4, d)}= & \frac{2}{3}\left(3 b_{6}^{F^{\prime}}\left(2 M_{K}^{2}+M_{\pi}^{2}\right)+b_{6}^{D^{\prime}}\left(2 M_{K}^{2}+M_{\pi}^{2}\right)\right. \\
& \left.+2\left(\beta_{1}\left(M_{\pi}^{2}-M_{K}^{2}\right)+3 \alpha_{2} M_{\pi}^{2}+\alpha_{4} M_{\pi}^{2}\right)\right), \\
\mu_{\Sigma^{-}}^{(4, d)}= & \frac{2}{3}\left(-3 b_{6}^{F^{\prime}}\left(2 M_{K}^{2}+M_{\pi}^{2}\right)+b_{6}^{D^{\prime}}\left(2 M_{K}^{2}+M_{\pi}^{2}\right)\right. \\
& \left.-2 \beta_{1} M_{K}^{2}-6 \alpha_{2} M_{\pi}^{2}+2 \alpha_{4} M_{\pi}^{2}+2 \beta_{1} M_{\pi}^{2}\right),
\end{aligned}
$$

$$
\begin{aligned}
\mu_{\Sigma^{0}}^{(4, d)}= & \frac{2}{3}\left(b_{6}^{D^{\prime}}\left(2 M_{K}^{2}+M_{\pi}^{2}\right)+2\left(\beta_{1}\left(M_{\pi}^{2}-M_{K}^{2}\right)+\alpha_{4} M_{\pi}^{2}\right)\right), \\
\mu_{\Lambda \Sigma^{0}}^{(4, d)}= & \frac{2}{\sqrt{3}}\left(b_{6}^{D^{\prime}}\left(2 M_{K}^{2}+M_{\pi}^{2}\right)+2 \alpha_{4} M_{\pi}^{2}\right), \\
\mu_{\Xi^{-}}^{(4, d)}= & -\frac{2}{3}\left(3 b_{6}^{F^{\prime}}\left(2 M_{K}^{2}+M_{\pi}^{2}\right)-b_{6}^{D^{\prime}}\left(2 M_{K}^{2}+M_{\pi}^{2}\right)\right. \\
& -2\left(-3 \alpha_{2} M_{K}^{2}-\alpha_{3} M_{K}^{2}+\alpha_{4} M_{K}^{2}-\beta_{1} M_{K}^{2}\right. \\
& \left.\left.+3 \alpha_{1}\left(M_{K}^{2}-M_{\pi}^{2}\right)+\alpha_{3} M_{\pi}^{2}+\beta_{1} M_{\pi}^{2}\right)\right), \\
\mu_{\Xi^{0}}^{(4, d)}= & -\frac{4}{3}\left(b_{6}^{D^{\prime}}\left(2 M_{K}^{2}+M_{\pi}^{2}\right)+2 \alpha_{4} M_{K}^{2}+\beta_{1} M_{K}^{2}\right. \\
& \left.-2 \alpha_{3}\left(M_{K}^{2}-M_{\pi}^{2}\right)-\beta_{1} M_{\pi}^{2}\right) .
\end{aligned}
$$

\section{B. Coefficients appearing in the NNLO loop contributions}

Here, we list the coefficients appearing in the loop contributions at NNLO.

$$
\begin{aligned}
& C_{p \pi}^{(4, e)}=-\frac{1}{2}\left(b_{6}^{F}+b_{6}^{D}\right), \\
& C_{p K}^{(4, e)}=-b_{6}^{F}, C_{n \pi}^{(4, e)}=\frac{1}{2}\left(b_{6}^{D}+b_{6}^{F}\right), C_{n K}^{(4, e)}=\frac{1}{2}\left(b_{6}^{D}-b_{6}^{F}\right), \\
& C_{\Lambda \pi}^{(4, e)}=0, \quad C_{\Lambda K}^{(4, e)}=\frac{1}{2} b_{6}^{D}, \\
& C_{\Sigma^{+} \pi}^{(4, e)}=-b_{6}^{F}, \quad C_{\Sigma^{+} K}^{(4, e)}=-\frac{1}{2}\left(b_{6}^{D}+b_{6}^{F}\right), \\
& C_{\Sigma^{-} \pi}^{(4, e)}=b_{6}^{F}, \\
& C_{\Sigma^{-} K}^{(4, e)}=\frac{1}{2}\left(b_{6}^{F}-b_{6}^{D}\right), C_{\Sigma^{0} \pi}^{(4, e)}=0, C_{\Sigma^{0} K}^{(4, e)}=-\frac{1}{2} b_{6}^{D}, \\
& C_{\Xi^{-} \pi}^{(4, e)}=\frac{1}{2}\left(b_{6}^{F}-b_{6}^{D}\right), \\
& C_{\Xi^{-} K}^{(4, e)}=b_{6}^{F}, \quad C_{\Xi^{0} \pi}^{(4, e)}=\frac{1}{2}\left(b_{6}^{D}-b_{6}^{F}\right), C_{\Xi^{0} K}^{(4, e)}=\frac{1}{2}\left(b_{6}^{D}+b_{6}^{F}\right), \\
& C_{\Lambda \Sigma^{0} \pi}^{(4, e)}=-\frac{1}{\sqrt{3}} b_{6}^{D}, C_{\Lambda \Sigma K}^{(4, e)}=-\frac{1}{2 \sqrt{3}} b_{6}^{D} .
\end{aligned}
$$

and

$$
\begin{aligned}
& C_{p \pi}^{(4, f)}=-\frac{1}{4}(D+F)^{2}\left(b_{6}^{D}-b_{6}^{F}\right), \\
& C_{p K}^{(4, f)}=\frac{1}{2}\left(\left(\frac{D^{2}}{9}-2 D F+F^{2}\right) b_{6}^{D}+(D-F)^{2} b_{6}^{F}\right), \\
& C_{p \eta}^{(4, f)}=\frac{1}{36}(D-3 F)^{2}\left(b_{6}^{D}+3 b_{6}^{F}\right), C_{n \pi}^{(4, f)}=\frac{1}{2}(D+F)^{2} b_{6}^{F}, \\
& C_{n K}^{(4, f)}=-\frac{1}{2}\left(\left(-\frac{7}{9} D^{2}+\frac{2}{3} D F+F^{2}\right) b_{6}^{D}+(D-F)^{2} b_{6}^{F}\right), \\
& C_{n \eta}^{(4, f)}=-\frac{1}{18}(D-3 F)^{2} b_{6}^{D}, \\
& C_{\Lambda \pi}^{(4, f)}=\frac{1}{3} D^{2} b_{6}^{D}, C_{\Lambda K}^{(4, f)}=-\frac{1}{18}\left(D^{2}+9 F^{2}\right) b_{6}^{D}+D F b_{6}^{F}, \\
& C_{\Lambda \eta}^{(4, f)}=-\frac{1}{9} D^{2} b_{6}^{D} \\
& C_{\Sigma^{+} \pi}^{(4, f)}=-\frac{1}{9}\left(D^{2}+6 D F-6 F^{2}\right) b_{6}^{D}+F^{2} b_{6}^{F}, \\
& C_{\Sigma^{+} K}^{(4, f)}=-\frac{1}{6}\left(D^{2}+6 D F+F^{2}\right) b_{6}^{D}+\frac{1}{2}(D-F)^{2} b_{6}^{F},
\end{aligned}
$$




$$
\begin{aligned}
C_{\Sigma^{+} \eta}^{(4, f)} & =\frac{1}{9} D^{2}\left(b_{6}^{D}+3 b_{6}^{F}\right), \\
C_{\Sigma^{-} \pi}^{(4, f)} & =-\frac{1}{9}\left(D^{2}-6 D F-6 F^{2}\right) b_{6}^{D}-F^{2} b_{6}^{F}, \\
C_{\Sigma^{-} K}^{(4, f)} & =-\frac{1}{6}\left(D^{2}-6 D F+F^{2}\right) b_{6}^{D}-\frac{1}{2}(D+F)^{2} b_{6}^{F}, \\
C_{\Sigma^{-} \eta}^{(4, f)} & =\frac{1}{9} D^{2}\left(b_{6}^{D}-3 b_{6}^{F}\right), \\
C_{\Sigma^{0} \pi}^{(4, f)} & =-\frac{1}{9}\left(D^{2}-6 F^{2}\right) b_{6}^{D}, \\
C_{\Sigma^{0} K}^{(4, f)} & =-\frac{1}{6}\left(D^{2}+F^{2}\right) b_{6}^{D}-D F b_{6}^{F}, \\
C_{\Sigma^{0} \eta}^{(4, f)} & =\frac{1}{9} D^{2} b_{6}^{D}, C_{\Xi^{-} \pi}^{(4, f)}=-\frac{1}{4}(D-F)^{2}\left(b_{6}^{D}+b_{6}^{F}\right), \\
C_{\Xi^{-} K}^{(4, f)} & =\frac{1}{2}\left(\frac{1}{9} D^{2}+2 D F+F^{2}\right) b_{6}^{D}-\frac{1}{2}(D+F)^{2} b_{6}^{F}, \\
C_{\Xi^{-} \eta}^{(4, f)} & =\frac{1}{36}(D+3 F)^{2}\left(b_{6}^{D}-3 b_{6}^{F}\right), \\
C_{\Xi^{0} \pi}^{(4, f)} & =-\frac{1}{2}(D-F)^{2} b_{6}^{F}, \\
C_{\Xi^{0} K}^{(4, f)} & =-\frac{1}{2}\left(-\frac{7}{9} D^{2}-\frac{2}{3} D F+F^{2}\right) b_{6}^{D}+\frac{1}{2}(D+F)^{2} b_{6}^{F}, \\
C_{\Xi^{0} \eta}^{(4, f)} & =-\frac{1}{18}(D+3 F)^{2} b_{6}^{D}, \\
C_{\Lambda \Sigma^{0} \pi}^{(4, f)} & =\frac{1}{3 \sqrt{3}}\left(D^{2} b_{6}^{D}-6 D F b_{6}^{F}\right), \\
C_{\Lambda \Sigma^{0} K}^{(4, f)} & =\frac{1}{2 \sqrt{3}}\left(3 F^{2}-D^{2}\right) b_{6}^{D}-\frac{1}{\sqrt{3}} D F b_{6}^{F}, \\
C_{\Lambda \Sigma^{0} \eta}^{(4, f)} & =-\frac{1}{3 \sqrt{3}} D^{2} b_{6}^{D} .
\end{aligned}
$$

The wave function renormalization coefficients are invariant under SU(2) transformations, therefore we give only the values of the different multiplets

$$
\begin{aligned}
& C_{N \pi}^{(4, g)}=\frac{3}{4}(D+F)^{2}, C_{N K}^{(4, g)}=\frac{5}{6} D^{2}-D F+\frac{3}{2} F^{2}, \\
& C_{N \eta}^{(4, g)}=\frac{1}{12}(D-3 F)^{2}, \\
& C_{\Sigma \pi}^{(4, g)}=\frac{1}{3} D^{2}+2 F^{2}, \quad C_{\Sigma K}^{(4, g)}=D^{2}+F^{2}, \\
& C_{\Sigma \eta}^{(4, g)}=\frac{1}{3} D^{2}, \\
& C_{\Lambda \pi}^{(4, g)}=D^{2}, C_{\Lambda K}^{(4, g)}=\frac{1}{3} D^{2}+3 F^{2}, C_{\Lambda \eta}^{(4, g)}=\frac{1}{3} D^{2}, \\
& C_{\Xi \pi}^{(4, g)}=\frac{3}{4}(D-F)^{2}, \\
& C_{\Xi K}^{(4, g)}=\frac{5}{6} D^{2}+D F+\frac{3}{2} F^{2}, \quad C_{\Xi \eta}^{(4, g)}=\frac{1}{12}(D+3 F)^{2} .
\end{aligned}
$$

and

$$
\begin{aligned}
& C_{p \pi}^{(4, h)}=2\left(b_{10}+b_{11}\right), \quad C_{p K}^{(4, h)}=b_{9}+4 b_{11}, \\
& C_{n \pi}^{(4, h)}=-2\left(b_{10}+b_{11}\right), \quad C_{n K}^{(4, h)}=-2 b_{10}+2 b_{11}, \\
& C_{\Lambda \pi}^{(4, h)}=0, \quad C_{\Lambda K}^{(4, h)}=-2 b_{10}, \quad C_{\Sigma^{+} \pi}^{(4, h)}=b_{9}+4 b_{11},
\end{aligned}
$$

$$
\begin{aligned}
& C_{\Sigma^{+} K}^{(4, h)}=2\left(b_{10}+b_{11}\right), \\
& C_{\Sigma^{-} \pi}^{(4, h)}=-b_{9}-4 b_{11}, \quad C_{\Sigma^{-} K}^{(4, h)}=2\left(b_{10}-b_{11}\right), \\
& C_{\Sigma^{0} \pi}^{(4, h)}=0, \quad C_{\Sigma^{0} K}^{(4, h)}=2 b_{10}, \\
& C_{\Xi^{-} \pi}^{(4, h)}=2\left(b_{10}-b_{11}\right), \quad C_{\Xi^{-} K}^{(4, h)}=-b_{9}-4 b_{11}, \\
& C_{\Xi^{0} \pi}^{(4, h)}=-2 b_{10}+2 b_{11}, \\
& C_{\Xi^{0} K}^{(4, h)}=-2\left(b_{10}+b_{11}\right), \quad C_{\Lambda \Sigma^{0} \pi}^{(4, h)}=\frac{4}{\sqrt{3}} b_{10}, \\
& C_{\Lambda \Sigma^{0} K}^{(4, h)}=\frac{2}{\sqrt{3}} b_{10} .
\end{aligned}
$$

and

$$
\begin{aligned}
& C_{p \pi}^{(4, i)}=-(D+F)^{2}\left(b_{D} M_{K}^{2}+b_{F}\left(M_{\pi}^{2}-M_{K}^{2}\right)\right), \\
& C_{p K}^{(4, i)}=-2 b_{D} M_{\pi}^{2}(D-F)^{2}, \\
& C_{p \eta}^{(4, i)}=-\frac{1}{3}(D-3 F)^{2}\left(b_{D} M_{K}^{2}+b_{F}\left(M_{\pi}^{2}-M_{K}^{2}\right)\right), \\
& C_{n \pi}^{(4, i)}=-2(D+F)^{2}\left(M_{K}^{2}\left(b_{D}-b_{F}\right)+b_{F} M_{\pi}^{2}\right), \\
& C_{n K}^{(4, i)}=2 b_{D} M_{\pi}^{2}(D-F)^{2}, C_{n \eta}^{(4, i)}=0, \\
& C_{\Lambda \pi}^{(4, i)}=0, \quad C_{\Lambda K}^{(4, i)}=-\frac{2}{3}\left(6 b_{D} D F M_{K}^{2}-b_{F}\left(D^{2}+9 F^{2}\right)\right. \\
& \left.\times\left(M_{K}-M_{\pi}\right)\left(M_{K}+M_{\pi}\right)\right), C_{\Lambda \eta}^{(4, i)}=0, \\
& C_{\Sigma^{+} \pi}^{(4, i)}=-4 b_{D} F^{2} M_{\pi}^{2}, C_{\Sigma^{+} K}^{(4, i)}=-2(D-F)^{2} \\
& \times\left(M_{K}^{2}\left(b_{D}-b_{F}\right)+b_{F} M_{\pi}^{2}\right) \text {, } \\
& C_{\Sigma^{+} \eta}^{(4, i)}=-\frac{4}{3} b_{D} D^{2} M_{\pi}^{2} \\
& C_{\Sigma^{-} \pi}^{(4, i)}=4 b_{D} F^{2} M_{\pi^{2}}^{2}, C_{\Sigma^{-} K}^{(4, i)}=2(D+F)^{2} \\
& \times\left(M_{K}^{2}\left(b_{D}+b_{F}\right)-b_{F} M_{\pi}^{2}\right), C_{\Sigma^{-} \eta}^{(4, i)}=\frac{4}{3} b_{D} D^{2} M_{\pi}^{2} \\
& C_{\Sigma^{0} \pi}^{(4, i)}=0, \\
& C_{\Sigma^{0} K}^{(4, i)}=2\left(2 b_{D} D F M_{K}^{2}+b_{F}\left(D^{2}+F^{2}\right)\right. \\
& \left.\times\left(M_{K}-M_{\pi}\right)\left(M_{K}+M_{\pi}\right)\right), C_{\Sigma^{0} K}^{(4, i)}=0, \\
& C_{\Xi^{-} \pi}^{(4, i)}=(D-F)^{2}\left(b_{D} M_{K}^{2}+b_{F}\left(M_{K}^{2}-M_{\pi}^{2}\right)\right), \\
& C_{\Xi^{-} K}^{(4, i)}=2 b_{D} M_{\pi}^{2}(D+F)^{2} \text {, } \\
& C_{\Xi^{-} \eta}^{(4, i)}=\frac{1}{3}(D+3 F)^{2}\left(b_{D} M_{K}^{2}+b_{F}\left(M_{K}^{2}-M_{\pi}^{2}\right)\right), \\
& C_{\Xi^{0} \pi}^{(4, i)}=2(D-F)^{2}\left(M_{K}^{2}\left(b_{D}+b_{F}\right)-b_{F} M_{\pi}^{2}\right), \\
& C_{\Xi^{0} K}^{(4, i)}=-2 b_{D} M_{\pi}^{2}(D+F)^{2} \text {, } \\
& C_{\Xi^{0} \eta}^{(4, i)}=0, \\
& C_{\Lambda \Sigma^{0} \pi}^{(4, i)}=8 / \sqrt{3} b_{D} D F M_{\pi}^{2}, \\
& C_{\Lambda \Sigma^{0} K}^{(4, i)}=2 / \sqrt{3}\left(2 b_{D} D F M_{K}^{2}-b_{F}\left(D^{2}-3 F^{2}\right)\left(M_{K}^{2}-M_{\pi}^{2}\right)\right) \text {, } \\
& C_{\Lambda \Sigma^{0} \eta}^{(4, i)}=0 \text {. }
\end{aligned}
$$

and

$$
\begin{aligned}
& C_{p \pi}^{(4, j)}=2(D+F)^{2}\left(M_{K}^{2} b_{D}+\left(M_{\pi}^{2}-M_{K}^{2}\right) b_{F}\right), \\
& C_{p K}^{(4, j)}=\frac{1}{3}\left((3 F+D)^{2} M_{\eta}^{2}+3(D-F)^{2} M_{\pi}^{2}\right) b_{D},
\end{aligned}
$$




$$
\begin{aligned}
C_{n \pi}^{(4, j)}= & -2(D+F)^{2}\left(M_{K}^{2} b_{D}+\left(M_{\pi}^{2}-M_{K}^{2}\right) b_{F}\right), \\
C_{n K}^{(4, j)}= & 2(D-F)^{2} M_{\pi}^{2} b_{D}, \\
C_{\Lambda \pi}^{(4, j)}= & 0, C_{\Lambda K}^{(4, j)}=-4 D F M_{K}^{2} b_{D} \\
& +\frac{2}{3}\left(9 F^{2}+D^{2}\right)\left(M_{K}^{2}-M_{\pi}^{2}\right) b_{F}, \\
C_{\Sigma^{+} \pi}^{(4, j)}= & \frac{4}{3}\left(3 F^{2} M_{\pi}^{2}+D^{2} M_{\eta}^{2}\right) b_{D}, \\
C_{\Sigma^{+} K}^{(4, j)}= & 2(D+F)^{2}\left(M_{K}^{2} b_{D}-\left(M_{\pi}^{2}-M_{K}^{2}\right) b_{F}\right), \\
C_{\Sigma^{-} \pi}^{(4, j)}= & -\frac{4}{3}\left(3 F^{2} M_{\pi}^{2}+D^{2} M_{\eta}^{2}\right) b_{D}, \\
C_{\Sigma^{-} K}^{(4, j)}= & -2(D-F)^{2}\left(M_{K}^{2} b_{D}+\left(M_{\pi}^{2}-M_{K}^{2}\right) b_{F}\right), \\
C_{\Sigma^{0} \pi}^{(4, j)}= & 0, C_{\Sigma^{0} K}^{(4, j)}=4 D F M_{K}^{2} b_{D} \\
& +2\left(D^{2}+F^{2}\right)\left(M_{K}^{2}-M_{\pi}^{2}\right) b_{F}, \\
C_{\Xi^{-} \pi}^{(4, j)}= & -2(D-F)^{2}\left(M_{K}^{2} b_{D}-\left(M_{\pi}^{2}-M_{K}^{2}\right) b_{F}\right), \\
C_{\Xi^{-} K}^{(4, j)}= & -\frac{4}{9} b_{D}\left(M_{K}^{2}(D-3 F)^{2}+2 D M_{\pi}^{2}(D+3 F)\right), \\
C_{\Xi^{0} \pi}^{(4, j)}= & 2(D-F)^{2}\left(M_{K}^{2} b_{D}+\left(M_{K}^{2}-M_{\pi}^{2}\right) b_{F}\right), \\
C_{\Xi^{0} K}^{(4, j)}= & -2(D-F)^{2} M_{\pi}^{2} b_{D}, \\
C_{\Lambda \Sigma^{0} \pi}^{(4, j)}= & 8 / \sqrt{3} D F M_{\pi}^{2} b_{D}, \\
C_{\Lambda \Sigma^{0} \pi}^{(4, j)}= & 4 / \sqrt{3} D F M_{K}^{2} b_{D} \\
& -2 / \sqrt{3}\left(3 F^{2}-D^{2}\right)\left(M_{\pi}^{2}-M_{K}^{2}\right) b_{F} . \\
&
\end{aligned}
$$

\section{References}

1. S.R. Coleman, S.L. Glashow, Phys. Rev. Lett. 6, 423 (1961)

2. J. Gasser, H. Leutwyler, Nucl. Phys. B 250, 465 (1985)

3. J. Gasser, M.E. Sainio, A. Svarc, Nucl. Phys. B 307, 779 (1988)

4. V. Bernard, N. Kaiser, U.-G. Meißner, Int. J. Mod. Phys. E 4, 193 (1995)
5. S. Scherer, Adv. Nucl. Phys. 27, 277 (2003)

6. V. Bernard, Prog. Part. Nucl. Phys. 60, 82 (2008)

7. D.G. Caldi, H. Pagels, Phys. Rev. D 10, 3739 (1974)

8. J. Bijnens, H. Sonoda, M.B. Wise, Nucl. Phys. B 261, 185 (1985)

9. E.E. Jenkins, M.E. Luke, A.V. Manohar, M.J. Savage, Phys. Lett. B 302, 482 (1993) [Erratum-ibid. B 388 (1996) 866]

10. B. Kubis, U.-G. Meißner, Eur. Phys. J. C 18, 747 (2001)

11. J.F. Donoghue, B.R. Holstein, T. Huber, A. Ross, Fizika B 14, 217 (2005)

12. L.S. Geng, J Martin Camalich, L. Alvarez-Ruso, M.J. Vicente Vacas, Phys. Rev. Lett. 101, 222002 (2008)

13. J. Gegelia, G. Japaridze, Phys. Rev. D 60, 114038 (1999)

14. T. Fuchs, J. Gegelia, G. Japaridze, S. Scherer, Phys. Rev. D 68, 056005 (2003)

15. L.S. Geng, J. Martin Camalich, M.J. Vicente Vacas, Phys. Lett. B 676, 63 (2009)

16. V. Bernard, H.W. Fearing, T.R. Hemmert, U.-G. Meißner, Nucl. Phys. A 635, 121 (1998) [Erratum: Nucl. Phys. A 642, 563 (1998)]

17. A.N. Hiller Blin, Phys. Rev. D 96, 093008 (2017)

18. L. Durand, P. Ha, Phys. Rev. D 58, 013010 (1998)

19. S.J. Puglia, M.J. Ramsey-Musolf, Phys. Rev. D 62, 034010 (2000)

20. U.-G. Meißner, S. Steininger, Nucl. Phys. B 499, 349 (1997)

21. E.E. Jenkins, A.V. Manohar, Phys. Lett. B 255, 558 (1991)

22. V. Bernard, N. Kaiser, J. Kambor, U.-G. Meißner, Nucl. Phys. B 388, 315 (1992)

23. T. Becher, H. Leutwyler, Eur. Phys. J. C 9, 643 (1999)

24. L.S. Geng, Front. Phys. (Beijing) 8, 328 (2013)

25. J.A. Oller, M. Verbeni, J. Prades, JHEP 0609, 079 (2006). arXiv:hep-ph/0608204

26. S.Z. Jiang, Q.S. Chen, Y.R. Liu, Phys. Rev. D 95, 014012 (2017)

27. T. Ledwig, J. Martin Camalich, L.S. Geng, M.J. Vicente Vacas, Phys. Rev. D 90(5), 054502 (2014). arXiv:1405.5456 [hep-ph]

28. S. Aoki et al., Eur. Phys. J. C 74, 2890 (2014). arXiv:1310.8555 [hep-lat]

29. S. Binder et al. (LENPIC Collaboration), Phys. Rev. C 93, 044002 (2016)

30. S. Boinepalli, D.B. Leinweber, A.G. Williams, J.M. Zanotti, J.B. Zhang, Phys. Rev. D 74, 093005 (2006)

31. T. Primer, W. Kamleh, D. Leinweber, M. Burkardt, Phys. Rev. D 89, $034508(2014)$

32. A. Parreno, M.J. Savage, B.C. Tiburzi, J. Wilhelm, E. Chang, W. Detmold, K. Orginos, Phys. Rev. D 95, 114513 (2017)

33. V. Pascalutsa, B.R. Holstein, M. Vanderhaeghen, Phys. Lett. B 600, 239 (2004) 